\title{
Basiliximab induction in kidney transplantation with donation after cardiac death donors
}

\author{
XUPING YAO, GUOBIN WENG, JUNJUN WEI and WENBO GAO \\ Department of Renal Transplantation, The Affiliated Urology and Nephrology Hospital, \\ College of Medicine, Ningbo University, Ningbo, Zhejiang 315100, P.R. China \\ Received January 12, 2015; Accepted February 18, 2016
}

DOI: $10.3892 /$ etm.2016.3238

\begin{abstract}
Basiliximab is a monoclonal antibody that binds to the $\alpha$-chain of the interleukin (IL)-2 receptor. It is used as induction therapy in kidney transplantation. The objective of the present study was to evaluate induction therapy with single-dose basiliximab (Simulect $\left.{ }^{\circledR}\right)$ in kidney transplantation with donation after cardiac death (DCD) donors. A total of 33 DCD kidney transplants were performed between December 2010 and July 2013 in patients who received single-dose basiliximab (20 mg) as induction therapy. The maintenance immunosuppression included calcineurin inhibitor (cyclosporine A or tacrolimus), mycophenolate mofetil and corticosteroids. The follow-up time was 1 year. The mean ages of the DCD donors and recipients were 29.3 and 41.1 years, respectively. Within the 1-year follow-up, the overall incidence of acute rejection was $9.1 \%$. There were 10 cases of delayed graft function among the recipients. Mean serum creatinine values at 1 week and at 1, 3, 6, 9 and 12 months post-transplantation were $257.6,238.2,194.5,159.3,137.9$ and $110.8 \mu \mathrm{mol} / 1$, respectively, with a favorable trend to allograft function recovery over time. The 1-year patient and graft survival rates were 96.9 and $90.9 \%$, respectively, with an infection rate of $24.2 \%$. Increased alanine aminotransferase/aspartate transaminase levels in only 2 patients were considered to be associated with basiliximab. This experience with single-dose basiliximab for induction therapy in DCD kidney transplantation showed that favorable clinical outcomes were achieved in terms of graft survival and function within 1 year.
\end{abstract}

Correspondence to: Professor Wenbo Gao, Department of Renal Transplantation, The Affiliated Urology and Nephrology Hospital, College of Medicine, Ningbo University, 1 Qianhe Road, Ningbo, Zhejiang 315100, P.R. China

E-mail:wenbogaocn@163.com

Key words: basiliximab, donation after cardiac death, kidney, transplantation, immunosuppression

\section{Introduction}

During the past few decades, nondepleting monoclonal antibody agents specifically targeting activated $\mathrm{T}$ cells have been developed, which are intended to provide effective immunosuppression without the adverse effects of profound and aspecific $\mathrm{T}$ lymphocyte depletion that are associated with polyclonal antibody agents (1). Basiliximab (Simulect $\left.{ }^{\circledR}\right)$ is a high affinity, chimeric monoclonal antibody, with murine variable and human constant regions, directed against the $\alpha$-chain (also known as CD25 subunit) of the interleukin (IL)-2 receptor on T lymphocytes (2). The expression of CD25 is specific to activated T cells; thus, basiliximab selectively targets activated lymphocytes and does not affect resting cells (3). The chimerization of the murine antibody with human immunoglobulin G1 is associated with reduced immunogenicity.

Basiliximab has been widely used as an induction therapy in kidney transplantation, with a two-dose regimen of $20 \mathrm{mg}$ on days 0 and 4, respectively. However, pharmacokinetic studies have shown that the half-life of basiliximab is $7.2 \pm 3$.2 days in adults, suggesting that a shorter regimen might be effective as an induction therapy (4). Recently, a single-dose regimen of basiliximab has been described in cadaveric kidney transplantation, with good efficacy and safety (4).

The objective of the present study was to observe the efficacy and safety of induction immunosuppression with single-dose basiliximab in 33 patients with donation after cardiac death (DCD) kidney transplantation in a single hospital.

\section{Materials and methods}

Patients. In this retrospective study, the clinical data of 33 DCD kidney surgeries from 25 donors performed between December 2010 and July 2013 at the Affiliated Urology and Nephrology Hospital of Ningbo University (Ningbo, China) were analyzed.

The study protocol was approved by the hospital's Committee for Medical and Health Research Ethics. It was conducted in accordance with the ethical guidelines of the 1975 Declaration of Helsinki. All patients included provided consent for the use of their clinical data in research.

All donors were China Category III donors (DCD or brain death patients). Donors' data were obtained from The Chinese Red Cross (Beijing, China). 
The donor selection criteria were as follows: $<60$ years of age; terminal urine output $>30 \mathrm{ml} / \mathrm{h}$; normal kidney size and morphology; absence of pre-existing renal disease; no medical history of cancer; absence of untreated systemic infection; negative serologic tests for human immunodeficiency virus, syphilis or hepatitis B or C infection.

The surgical technique of organ procurement is briefly described as follows: The donor was transported to the operating room so that no time was lost in transportation and the warm ischemia time (WIT) was reduced. The patient was pretreated with an intravenous injection of 10,000 units heparin to decrease the risk of thrombosis. After withdrawal of life support and declaration of cardiac death, rapid laparotomy was conducted with direct cannulation of the aorta and in situ perfusion with UW solution (Bristol-Myers Squibb, New York, NY, USA). In all cases, abdominal organs were cooled using ice-slush. Then, multiple organ procurement was performed using conventional organ recovery techniques (5). Subsequently, the organs were rapidly flushed, cooled, and preserved in UW preservation solution.

Immunosuppressive regimen. Prior to this study, basiliximab (Novartis Pharmaceuticals Corp., East Hanover, NJ, USA) was used in induction therapy in conventional two doses (days 0 and 4 post-transplantation). However, following careful observation and an accumulation of clinical experience, in 2006, the induction protocol was changed and a single-dose basiliximab protocol was introduced.

In this study, for all the DCD kidney recipients, a bolus of $20 \mathrm{mg}$ basiliximab was given $1 \mathrm{~h}$ prior to the surgery, lasting until after the completion of the vascular anastomoses. In addition, all recipients received $500 \mathrm{mg}$ intravenous methylprednisolone (Pfizer, Inc,, New York, NY, USA) during the surgery and a 3-day bolus of intravenous methylprednisolone therapy (10 mg/kg per day) post-transplantation. Subsequently, the patients received oral prednisone (Xianju Pharmaceutical Co., Ltd., Hangzhou, China) at $80 \mathrm{mg}$ per day, with further tapering to $20 \mathrm{mg}$ per day at 10 days, and $10 \mathrm{mg}$ per day at 3 to 4 months.

The standard immunosuppressive protocol for all kidney transplant recipients consisted of the following: A calcineurin inhibitor, such as cyclosporine A (CsA; Neoral ${ }^{\circledR}$; Novartis, Basel, Switzerland) or tacrolimus (Astellas Pharma, Deerfield, IL, USA); mycophenolate mofetil (MMF; Roche Pharmaceuticals, Nutley, NJ, USA); and corticosteroids (prednisone). Initial target $\mathrm{CsA}$ blood concentration at $2 \mathrm{~h}$ after dose (C2) levels were $900-1,100 \mathrm{ng} / \mathrm{ml}$, tapering to $800-1,000 \mathrm{ng} / \mathrm{ml}$ by 2 months and thereafter CsA C2 levels were $600-800 \mathrm{ng} / \mathrm{ml}$ with gradual reduction towards a CsA C2 of 500-600 ng/ml in the long-term maintenance phase. Initial tacrolimus trough levels were targeted at $8-10 \mathrm{ng} / \mathrm{ml}$ in the first 3 months, while subsequent doses were adjusted to $6-8 \mathrm{ng} / \mathrm{ml}$ thereafter. For mycophenolate mofetil, a dose of $1,000 \mathrm{mg}$ twice daily was given at the time of transplantation, lasting for 1 week; then the dose was adjusted to $750 \mathrm{mg}$ twice daily for 1 week, and a further reduction to $500 \mathrm{mg}$ twice daily continued thereafter.

Diagnosis of acute rejection. In this study, the incidence and severity (determined by histological grade) of acute rejection during the post-transplant period were observed. Acute rejections were diagnosed by persistent increase of serum creatinine ( $\mathrm{Cr}$; by $\geq 15 \%$ from the baseline) not explained by CsA or tacrolimus levels above the target levels or by kidney hypoperfusion, vascular thrombosis, or urinary tract obstruction; clinical signs of rejection including fever $\left(>38.0^{\circ} \mathrm{C}\right)$, decreased urinary output, pain over an enlarged kidney graft, hypertension, an increased kidney graft size indicated by color Doppler and elevated vascular resistance index (>0.8). Acute rejection was confirmed by biopsy. Biopsies obtained were graded according to the updated Banff Classification (6).

Prophylaxis of infection. All patients received perioperative ceftriaxone for bacterial prophylaxis. Two weeks after the surgery, all recipients received anti-Pneumocystis prophylaxis with sulfamethoxazole/trimethoprim for 3 months. Antiviral prophylaxis consisted of intravenous ganciclovir for $\geq 1$ month, depending on the donor and recipient cytomegalovirus (CMV) serologic status.

Patient monitoring and follow-up. Post-transplantation renal allograft function was evaluated by measuring serum $\mathrm{Cr}$ levels as well as $\mathrm{Cr}$ clearance. In all patients, routine clinical and laboratory parameters, including serum $\mathrm{Cr}$ concentration, red blood cell, white blood cell (WBC) and platelet counts, were monitored daily up to post-transplant day 14 while hospitalized, then followed up at regular intervals following discharge. Furthermore, patient and graft survivals, and the incidence of delayed graft function (DGF) were also observed. DGF was defined as a requirement for dialysis treatment within 1 week after transplantation.

After discharge from the hospital, the patients were followed up at the out-patient clinic on a weekly basis during the first 3 months, every 2 weeks until month 6 , and then monthly until the end of the first post-transplant year. On every visit, complete blood counts, serum $\mathrm{Cr}$ concentration, and urine examination results were monitored and recorded on a database. Color Doppler studies were performed as per requirement, and additional evaluations were performed whenever deemed clinically appropriate. The follow-up period was 12 months.

Safety assessment. Safety was assessed by monitoring adverse events during the course of the study, including the incidence and type of possible side effects associated with basiliximab. In particular, the incidence and severity of infections, malignancy and abnormal results of laboratory tests were monitored.

Statistical analysis. All data are expressed as mean \pm standard deviation. The SPSS 16.0 statistical software package (SPSS, Inc., Chicago, IL, USA) was used.

\section{Results}

Patient characteristics. Characteristics of DCD donors and recipients are summarized in Tables I and II, respectively.

The mean age of the DCD donors was 29.3 years (range, 2-53 years; Table I). There were 15 male and 10 female donors. The mean WIT was 13.9 min, with a mean cold ischemia time of $187.4 \mathrm{~min}$. The causes of mortality included traumatic brain injury (20 cases, $80.0 \%$ ), drowning (3 cases, $12.0 \%$ ) and other 
Table I. Demographic and clinical characteristics of donors after cardiac death.

\begin{tabular}{lc}
\hline Characteristic & Donors $(\mathrm{n}=25)$ \\
\hline Age, years & \\
Mean ${ }^{\mathrm{a}}$ & $29.3 \pm 14.5$ \\
Range & $2-53$ \\
Gender & \\
Male & 15 \\
Female & 10 \\
sCr concentration on the day & $150.7 \pm 31.9$ \\
of transplantation, $\mu{\text { mol/ } / 1^{\mathrm{a}}}^{\mathrm{a}}$ & \\
Warm ischemia time, min $^{\mathrm{a}}$ & $13.9 \pm 6.2$ \\
Cold ischemia time, min $^{\mathrm{a}}$ & $187.4 \pm 33.7$ \\
Cause of death, $\mathrm{n}$ & \\
Traumatic brain injury & 20 \\
Drowning & 3 \\
Others & 2 \\
\hline
\end{tabular}

${ }^{\text {a } V a l u e s}$ are presented as mean \pm standard deviation; $\mathrm{sCr}$, serum creatinine.

reasons ( 1 case of ganglia glioma and 1 case of Guillian-Barre syndrome, $8.0 \%)$. The serum $\mathrm{Cr}(\mathrm{sCr})$ level prior to organ procurement was $150.7 \pm 31.9 \mu \mathrm{mol} / \mathrm{l}$. Among the donors, the paired kidneys from one 2-year-old donor were en bloc engrafted into one 14-year-old female recipient.

The 33 kidney recipients had a mean age of 41.1 years (range, 2-63 years; Table II), and included 19 males and 14 females. Original diseases leading to end stage renal disease (ESRD) were chronic glomerulonephritis $(n=18)$, hypertension $(n=7)$, diabetic nephropathy $(n=6)$ and polycystic kidney $(n=2)$. All the recipients had a panel reactive antibody (PRA) value of $<10 \%$, with a mean of $4.1 \%$. In addition, the proportion for human leukocyte antigen (HLA) mismatch at 1, 2, 3, 4, 5 and 6 loci was $6.1,15.2,48.4,18.2,9.1$ and $3.0 \%$, respectively. None of the recipients presented anti-HLA antibodies.

Acute rejection episodes (AREs). At 1 year post-transplantation, there were a total of 3 AREs observed in 3 patients, with an overall incidence of $9.1 \%$. Renal biopsy confirmed the diagnosis of rejection in these patients. Among them, 2 patients had grade I rejection; and in 1 case, acute rejection was graded as II. All patients with acute rejection were treated with a combination of intravenous administration of methylprednisolone pulses $(8 \mathrm{mg} / \mathrm{kg}$ per day) and cyclophosphamide (CTX, $0.2 \mathrm{~g}$ per day) for 3 days followed by a steroid taper. The majority of AREs responded to this therapy, and only 1 patient was treated with anti-thyroglobulin (ATG) antibodies ( $2 \mathrm{mg} / \mathrm{kg}$ per day intravenously) for 7 days for steroid-resistant ARE. All patients with AREs were eventually reversed and there were no recurrences.

Recovery of allograft function. No patients experienced hyperacute rejection reaction. There were 10 cases of DGF in the recipients, with an incidence of $30.3 \%$. One patient with DGF
Table II. Demographic and clinical characteristics of recipients.

Characteristic

Recipients (n=33)

Age, years

Mean $^{\mathrm{a}}$

Range

$41.1 \pm 15.7$

$12-63$

Gender

Male

19

Female

Weight ratio (graft-to-recipient), $\mathrm{g} / \mathrm{kg}^{\mathrm{a}}$

$2.8 \pm 0.7$

Underlying kidney disease, $\mathrm{n}$

Chronic glomerulonephritis

18

Hypertension

7

Diabetes

Polycystic kidney

6

2

Mode of pretransplantation dialysis, $n$

Hemodialysis

26

Peritoneal dialysis

7

Duration of pretransplantation dialysis, $\min ^{\mathrm{a}}$

$10.6 \pm 4.5$

PRA, \% ${ }^{\mathrm{a}}$

$4.1 \pm 2.5$

sCr clearance, $\mathrm{ml} / \mathrm{min}^{\mathrm{a}}$

$\begin{array}{ll}1 \text { month } & 37.9 \pm 12.5 \\ 6 \text { month } & 56.7 \pm 16.1 \\ 12 \text { month } & 70.4 \pm 20.6\end{array}$

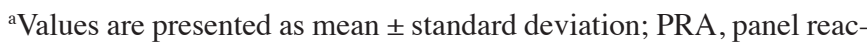
tive antibody; sCR, serum creatinine.

developed severe bleeding from the renal vein leading to graft loss at 14 days post-transplantation due to Mycosis pilorum infection. Others $(9 / 10,90.0 \%)$ recovered renal graft function to normal levels within 9-32 days (13.3 \pm 6.5 days): $66.7 \%(6 / 9)$ by 14 days and $33.3 \%$ (3/9) by 32 days.

The mean $\mathrm{sCr}$ values at 1 week, and at 1, 3, 6, 9 and 12 months post-transplantation were $257.6 \pm 89.3,238.2 \pm 74.1$, $194.5 \pm 57.5,159.3 \pm 48.6,137.9 \pm 40.2$ and $110.8 \pm 32.9 \mu \mathrm{mol} / 1$, respectively. These results showed a favorable trend to allograft function recovery over time (Fig. 1).

Patient and allograft survival. By the end of the follow-up, 1 patient succumbed due to Guillian-Barre syndrome at 3 months post-transplantation; at the time of death, the transplant was functioning with a plasma $\mathrm{Cr}$ of $127 \mu \mathrm{mol}$. In addition, 1 patient experienced graft loss from severe pulmonary infection; and one graft was lost from severe bleeding of the renal vein due to Mycosis pilorum infection, as mentioned above. Overall, 1-year patient and graft survival rates were 96.9 and $90.9 \%$, respectively. Furthermore, when reduction of $\mathrm{sCr}$ to $<177.0 \mu \mathrm{mol} / 1$ was regarded as normalization of the renal allograft, the results demonstrated that the proportion of patients with normalization of the renal allograft gradually increased as the time elapsed, as shown in Fig. 2.

Safety analyses. During the follow-up period, no patient developed a malignancy. Infections occurred in 8 patients with an 


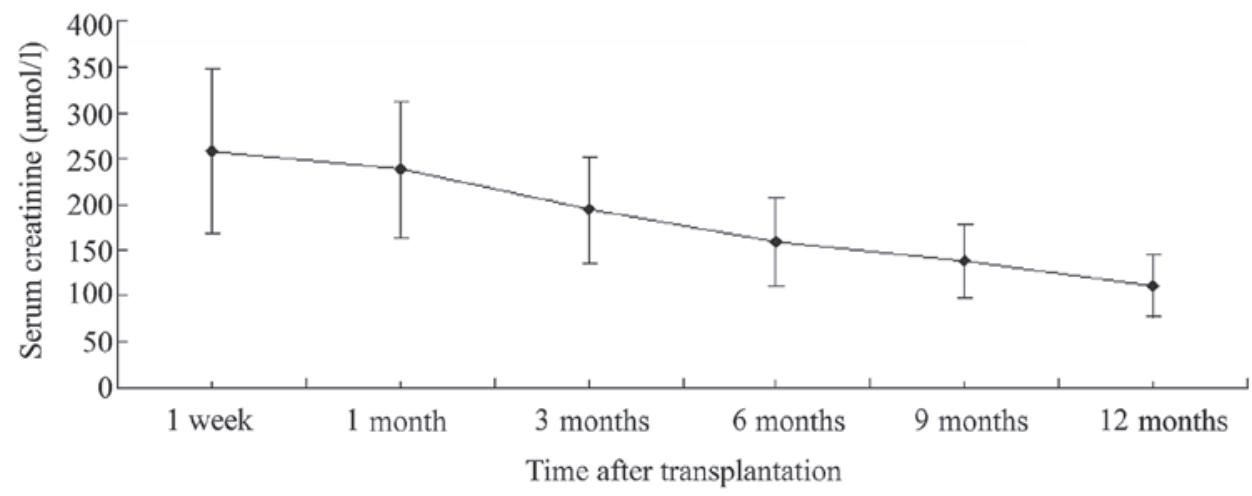

Figure 1. Changes of serum creatinine levels during the first post-transplantation year.

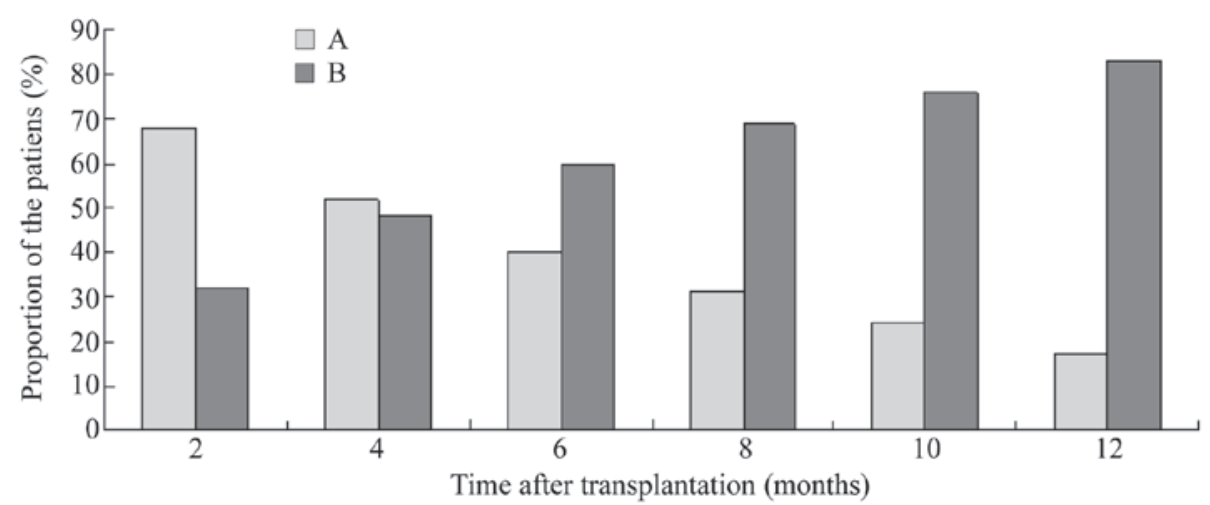

Figure 2. Proportion of the patients with serum creatinine levels of (A) $\geq 177.0$ or (B) $<177.0 \mu \mathrm{mol} / 1 \mathrm{in}$ the first post-transplantation year.

overall incidence of $24.2 \%$, including 6 cases of pulmonary infection, 1 case of urinary tract infection and 1 case of Mycosis pilorum infection in the renal vein. No CMV infection was observed. In addition, a total of 12 patients presented with other adverse events, including increasing alanine aminotransferase (ALT)/aspartate transaminase (AST) levels (>50 U/1) in 8 patients, hypertension $(>140 / 90 \mathrm{mmHg})$ in 6 patients and leukopenia (defined as WBC counts $<3,500 / \mu 1$ ) in 2 patients. However, following careful examination and further evaluation, increasing ALT/AST was considered to be associated with basiliximab in only 2 patients, and they were recovered following appropriate symptomatic treatment.

\section{Discussion}

Renal transplantation is the preferred treatment for the majority of patients with ESRD because transplantation markedly improves the length and quality of life for dialysis patients (7). At the end of 2013, according to data from the Chinese Renal Data Registration System, the number of patients in mainland China receiving dialysis was 326,000 (280,000 hemodialysis and 46,000 peritoneal dialysis patients), accounting for $\sim 20 \%$ of the global ESRD population; however, the donor supply for kidney transplantation in China is rather limited (8). The discrepancy between renal transplant supply and demand in China is expected to grow as the population ages, as the prevalence of hypertension, diabetes increases, and with the increasing number of patients whose previous allograft loses function. Allografts from DCD donors are increasingly being used (9). It has been reported that long-term results equivalent to those with deceased donors can be achieved using kidneys from DCD donors. To further improve these results, the use of induction therapy continues to be investigated in DCD kidney transplantation (10).

Induction therapy in kidney transplantation is considered to be important for reducing acute rejections, despite the potential for adverse effects. Induction therapies include polyclonal anti-lymphocyte antibodies, monoclonal anti-lymphocyte antibodies and anti-IL-2 receptor monoclonal antibody (7). The chimeric IL-2 receptor antibody basiliximab has been widely used as an effective, safe and economical induction therapy for kidney transplantation. Basiliximab is directed against the CD25 subunit, which prevents acute cellular rejection (ACR) by inhibiting IL-2-driven T-cell proliferation (11). Basiliximab is one of the most commonly used induction agents due to the following advantages: i) Evidence of reduced overall cost demonstrated by pharmacoeconomic analysis; ii) ease of administration; iii) short-term use; iv) no need for blood level monitoring; and v) lack of major toxicity.

Studies have shown that induction therapy with basiliximab leads to greater graft and patient survival rates $(12,13)$. In a large prospective placebo-controlled trial of basiliximab in 700 adult renal transplant patients in Europe, the result showed a significant reduction in rejection in the first year after transplantation, with a $32 \%$ reduction in biopsy-proven acute rejection in the first 6 months (from $44 \%$ with placebo to $30 \%$ with basiliximab) and a $57 \%$ reduction in steroid-resistant rejection with the use of basiliximab (1). 
Similarly, a large meta-analysis reported a rate of acute rejection of $40 \%$ in patients receiving placebo or no induction, with a reduction in the risk of acute rejection of $34 \%$ (relative risk, 0.66) using basiliximab (14). Thus, for every 100 patients treated with IL-2 receptor antagonist, it was expected that 14 fewer patients would experience acute rejection, and 7 patients would need to be treated to prevent one rejection episode [number needed to treat (NNT) acute rejection $=7]$. An updated analysis found a $38 \%$ incidence of rejection in the placebo/no induction arm, and suggested an NNT of 9 patients to prevent one episode of acute rejection (15).

In the present study, 33 renal transplantations from DCD donors using a single-dose basiliximab induction strategy were evaluated. The 1-year graft survival rate was $90.9 \%$, similar to that observed by Ledinh et al (16). An intraoperative 20-mg single-dose of basiliximab was used in the present study, which is lower than that used by Matl et al (4), who demonstrated that the efficacy, safety and tolerability of a 40-mg single-dose basiliximab regimen were comparable to those of the conventional 2 x $20 \mathrm{mg}$ two-dose regimen. Since Chinese people may be considered to have a smaller body size than westerners, this $20 \mathrm{mg}$ single-dose regimen of basiliximab in DCD kidney transplantation in Chinese patients is valid.

Infection is an important complication after transplantation that could affect the life quality and survival of recipients. Induction therapy in renal transplantation is prone to increase the risk for infection. In the present study, 8 patients developed infection, including 6 cases of pulmonary infection, 1 case of urinary tract infection and 1 case of Mycosis pilorum infection in the renal vein. This infection rate described in these DCD recipients is not specific to these particular patients but is typical of patients in the transplantation setting in China. Of note, for the recipient diagnosed with Mycosis pilorum infection in the renal vein, the donor was drowned in a muddy pool; and after further investigation, it was found that the recipient receiving the other kidney underwent graft nephrectomy for rupture and severe bleeding of the allograft in the third postoperative week (in another hospital). One patient succumbed to Guillain-Barré syndrome (GBS) at 3 months post-transplantation. This patient's HLA loci matched well with the donor who also died of GBS; they had only one HLA mismatch. The 36-year-old patient had excellent renal function after transplantation; but 10 weeks later, he was readmitted due to progressive, symmetrical weakness of the lower limbs, paresthesia and dysphagia, and in the next few days he developed acute respiratory failure requiring mechanical ventilation. At the time of his death, his transplant was functioning well. Our evaluation of infectious complications did not show a significant difference in incidence or its role as a cause of mortality/graft loss. These data are consistent with the meta-analysis of Adu et al (17).

During the study period, 10 patients experienced DGF, a common complication in the immediate post-transplant period, with an incidence of $30.3 \%$. The incidence of DGF after transplantation from DCD donors has been described as 25 to $90 \%$ (18). Several studies have documented that one of the potential problems with kidneys from DCD donors is the potential for a higher rejection rate and a higher risk for graft failure due to the higher incidence of DGF $(19,20)$. In addition, DGF leads to extended hospital stays, resulting in increased costs (19). The commonly accepted explanation for the increased risk of DGF in DCD kidney transplantation is warm ischemia, which results in reperfusion injury, acute tubular necrosis and graft dysfunction. The variable period of warm ischemia that occurs prior to organ procurement is a major disadvantage of DCD organ donation. While the interval from asystole to cross-clamp and organ perfusion is readily determined, warm ischemia occurring during the withdrawal phase prior to asystole is much more difficult to quantify (21). In the present study, the duration of warm ischemia was taken as being from the time of persistent systolic blood pressure $<60 \mathrm{mmHg}$ to the time of organ reperfusion with cold preservation solution. The outcome would be improved by any intervention that could preserve the function of the graft and reduce the incidence of DGF; improved methods for organ recovery and reduction of drug toxicity may, therefore, further improve the outcome of DCD kidney transplantation. Basiliximab has been reported to be associated with reduced DGF incidence, mainly when calcineurin inhibitor administration has been reduced or delayed to avoid nephrotoxicity to ischemic kidneys recovering from cold storage (22).

It has been reported that basiliximab does not cause lymphocyte depletion, with an incidence of cytopenia lower than that with depleting agents such as ATG or alemtuzumab, and partial humanization structurally decreases the development of inhibitory antibodies (23). It is administered peripherally and is less expensive than ATG in China. The incidence of infusion reactions is also lower with basiliximab than with depleting agents (24). In the present study cohort, increasing ALT/AST was considered to be associated with basiliximab in only 2 patients, and they both recovered satisfactorily. This clinical study demonstrated that the application of single-dose of basiliximab as an induction therapy in DCD kidney transplantation is very well tolerated.

The shortcomings of the present study include it being a single-center evaluation, with a relatively small population and short duration of study, as well there being as lack of comparison between single-dose and conventional two-dose basiliximab regimens in DCD kidney transplantation. Furthermore, in future studies, pre-operative evaluation of the match of the donor and recipient requires further improvement, including construction of better inclusion criteria and exclusion criteria, in order to reduce the number of mismatches between the donor and recipient.

The present study of single-dose of basiliximab as an induction therapy in DCD kidney transplantation showed that favorable clinical outcomes within 1 year were achieved in terms of graft survival and function. The single-dose regimen in a larger population as well as in comparison with a conventional two-dose regimen in DCD kidney transplant recipients requires further investigation.

\section{Acknowledgements}

The authors would like to thank Mr. Jianfeng Zhu (Department of Clinical Laboratory, Yinzhou No. 2 Hospital, Ningbo, China) for his excellent laboratory management. 


\section{References}

1. Clark G, Walsh G, Deshpande P and Koffman G: Improved efficacy of basiliximab over antilymphocyte globulin induction therapy in paediatric renal transplantation. Nephrol Dial Transplant 17: 1304-1309, 2002.

2. Ferrer F, Machado S, Alves R, Macário F, Bastos C, Roseiro A and Mota A: Induction with basiliximab in renal transplantation. Transplant Proc 42: 467-470, 2010.

3. Jorge S, Guerra J, Silva S, Santana A, Mil-Homens C and Prata MM: Induction immunosuppressive therapy in renal transplantation: Does basiliximab make the difference? Transplant Proc 40: 693-696, 2008.

4. Matl I, Bachleda P, Michalsky R, Navratil P, Lao M, Treska V, Prestele H, Matthisson M and Korn A: Basiliximab can be administered safely and effectively in a single dose on day 1 postrenal transplantation in patients receiving triple therapy with azathioprine. Transplant Proc 33: 3205-3206, 2001.

5. Gravel MT, Arenas JD, Chenault R II, Magee JC, Rudich S, Maraschio M, DebRoy M, Miller W and Punch JD: Kidney transplantation from organ donors following cardiopulmonary death using extracorporeal membrane oxygenation support. Ann Transplant 9: 57-58, 2004.

6. Solez K and Racusen LC: The Banff classification revisited. Kidney Int 83: 201-206, 2013.

7. Ocampo C, Aristizabal A, Nieto J, Abadia H, Angel W, Guzman C, Mena A, Vanegas J, Velez C, Aguirre C, et al: Induction therapies in kidney transplantation: The experience of hospital Pablo Tobon Uribe, Medellin, Colombia 2005-2010 Transplant Proc 43: 3359-3363, 2011.

8. Yu X and Yang X: Peritoneal dialysis in China: Meeting the challenge of chronic kidney failure. Am J Kidney Dis 65: 147-151, 2015.

9. Wind J, van Mook WN, Willems ME and van Heurn LW: Higher organ donation consent rates by relatives of potential uncontrolled donors versus potential controlled donors after death. Nephrol Dial Transplant 27: 4219-4223, 2012.

10. Vandivort CS, Taber D, McGillicuddy JW, Bratton C, Chavin K and Baliga P: Protocol based induction therapy improves rejection rates, complications and transplant event costs in adult kidney transplant recipients. J Surg Res 186: 655, 2014.

11. Naderi GH, Mehraban D, Ganji MR, Jafarpouriani M and Latif AH: The outcome of induction therapy with monoclonal antibodies in kidney transplantation among Iranian patients: A prospective study. Transplant Proc 41: 2768-2771, 2009.
12. Gralla $J$ and Wiseman AC: The impact of IL2ra induction therapy in kidney transplantation using tacrolimus- and mycophenolate-based immunosuppression. Transplantation 90: 639-644, 2010.

13. Heldal K, Thorarinsdottir S, Hartmann A,Leivestad T, Reisæter AV, Foss AE and Midtvedt K: Induction with interleukin-2 antagonist for transplantation of kidneys from older deceased donors: an observational study. Transplant Res 2: 2-11, 2013.

14. Liu Y, Zhou P, Han M, Xue CB, Hu XP and Li C: Basiliximab or antithymocyte globulin for induction therapy in kidney transplantation: A meta-analysis. Transplant Proc 42: 1667-1670, 2010

15. Webster AC, Playford EG, Higgins G, Chapman JR and Craig J: Interleukin 2 receptor antagonists for kidney transplant recipients. Cochrane Database Syst Rev: CD003897, 2004.

16. Ledinh H, Bonvoisin C, Weekers L, de Roover A, Honoré P, Squifflet JP, Meurisse M and Detry O: Results of kidney transplantation from donors after cardiac death. Transplant Proc 42: 2407-2414, 2010.

17. Adu D, Cockwell P, Ives NJ, Shaw J and Wheatley K: Interleukin-2 receptor monoclonal antibodies in renal transplantation: Meta-analysis of randomized trials. BMJ 326: 789, 2003.

18. Quiroga I, McShane P, Koo DD, Gray D, Friend PJ, Fuggle S and Darby C: Major effects of delayed graft function and cold ischaemia time on renal allograft survival. Nephrol Dial Transplant 21: 1689-1696, 2006.

19. Farney AC, Hines MH, al-Geizawi S, Rogers J and Stratta RJ: Lessons learned from a single center's experience with 134 donation after cardiac death donor kidney transplants. J Am Coll Surg 212: 440-451, 2011.

20. Snoeijs MGJ, Ernest van Heurn LW, van Mook WNKA, Christiaans MH and van Hooff JP: Controlled donation after cardiac death: A European perspective. Transplantation Reviews 21: 219-229, 2007.

21. Abt PL, Fisher CA and Singhal AK: Donation after cardiac death in the US: History and use. J Am Coll Surg 203: 208-225, 2006.

22. Wagner SJ and Brennan DC: Induction therapy in renal transplant recipients: How convincing is the current evidence? Drugs 72: 671-683, 2012.

23. Favi E, Gargiulo A, Spagnoletti G, Salerno MP, Silvestrini N, Valente I and Citterio F: Induction with basiliximab plus thymoglobulin is effective and safe in old-for-old renal transplantation: Six-month results of a prospective clinical study. Transplant Proc 42: 1114-1117, 2010.

24. Haririan A, Morawski K, Sillix DH, El-Amm JM, Garnick J, WestMS,GrangerDK,MigdalSDandGruberSA:Inductiontherapy with basiliximab versus thymoglobulin in African-American kidney transplant recipients. Transplantation 79: 716-721, 2005. 